\title{
HIERARCHICAL STRUCTURE OF THE FAMILY OF CURVES WITH MAXIMAL GENUS VERIFYING FLAG CONDITIONS
}

\author{
VINCENZO DI GENNARO
}

(Communicated by Michael Stillman)

\begin{abstract}
Fix integers $r, s_{1}, \ldots, s_{l}$ such that $1 \leq l \leq r-1$ and $s_{l} \geq r-l+1$, and let $\mathcal{C}\left(r ; s_{1}, \ldots, s_{l}\right)$ be the set of all integral, projective and nondegenerate curves $C$ of degree $s_{1}$ in the projective space $\mathbf{P}^{r}$, such that, for all $i=2, \ldots, l$, $C$ does not lie on any integral, projective and nondegenerate variety of dimension $i$ and degree $<s_{i}$. We say that a curve $C$ satisfies the flag condition $\left(r ; s_{1}, \ldots, s_{l}\right)$ if $C$ belongs to $\mathcal{C}\left(r ; s_{1}, \ldots, s_{l}\right)$. Define $G\left(r ; s_{1}, \ldots, s_{l}\right)=$ $\max \left\{p_{a}(C): C \in \mathcal{C}\left(r ; s_{1}, \ldots, s_{l}\right)\right\}$, where $p_{a}(C)$ denotes the arithmetic genus of $C$. In the present paper, under the hypothesis $s_{1} \gg \cdots \gg s_{l}$, we prove that a curve $C$ satisfying the flag condition $\left(r ; s_{1}, \ldots, s_{l}\right)$ and of maximal arithmetic genus $p_{a}(C)=G\left(r ; s_{1}, \ldots, s_{l}\right)$ must lie on a unique flag such as $C=V_{s_{1}}^{1} \subset V_{s_{2}}^{2} \subset \cdots \subset V_{s_{l}}^{l} \subset \mathbf{P}^{r}$, where, for any $i=1, \ldots, l, V_{s_{i}}^{i}$ denotes an integral projective subvariety of $\mathbf{P}^{r}$ of degree $s_{i}$ and dimension $i$, such that its general linear curve section satisfies the flag condition $\left(r-i+1 ; s_{i}, \ldots, s_{l}\right)$ and has maximal arithmetic genus $G\left(r-i+1 ; s_{i}, \ldots, s_{l}\right)$. This proves the existence of a sort of a hierarchical structure of the family of curves with maximal genus verifying flag conditions.
\end{abstract}

Fix integers $r, d, s$ such that $s \geq r-1$, and let $\mathcal{C}(r ; d, s)$ be the set of all integral, projective and nondegenerate curves of degree $d$ in the projective space $\mathbf{P}^{r}$, not contained in any integral, projective surface of degree $<s$. Extending the classical results of Halphen $[\mathrm{H}]$, Noether $[\mathrm{N}]$ and Castelnuovo $[\mathrm{C}]$, and more recent results of Gruson and Peskine [GP], and Eisenbud and Harris [EH], in [CCD] one proves that, when $d \gg s$, the curves of maximal arithmetic genus in $\mathcal{C}(r ; d, s)$ are contained in surfaces of degree $s$, whose general hyperplane sections are themselves curves of maximal arithmetic genus in $\mathcal{C}(r-1 ; s, r-2)$ (the so-called "Castelnuovo curves"). In the present paper we show that this property is a particular case of a more general property.

In order to state our main result, we need some preliminary notation. Fix integers $r, s_{1}, \ldots, s_{l}$ such that $1 \leq l \leq r-1$ and $s_{l} \geq r-l+1$, and let $\mathcal{C}\left(r ; s_{1}, \ldots, s_{l}\right)$ be the set of all integral, projective and nondegenerate curves $C$ of degree $s_{1}$ in the projective space $\mathbf{P}^{r}$, such that, for all $i=2, \ldots, l, C$ does not lie on any integral, projective and nondegenerate variety of dimension $i$ and degree $<s_{i}$. We say that a curve $C$ satisfies the flag condition $\left(r ; s_{1}, \ldots, s_{l}\right)$ if $C$ belongs to $\mathcal{C}\left(r ; s_{1}, \ldots, s_{l}\right)$. Notice that $\mathcal{C}\left(r ; s_{1}, r-1\right)$ is simply the set of all integral, projective and nondegenerate curves

Received by the editors April 21, 2005 and, in revised form, October 15, 2006.

2000 Mathematics Subject Classification. Primary 14N15, 14H99; Secondary 14N30, 14M05.

Key words and phrases. Complex projective curve, Castelnuovo-Halphen theory, arithmetically Cohen-Macaulay curve, arithmetic genus, flag condition, adjunction formula. 
of degree $s_{1}$ in $\mathbf{P}^{r}$ (i.e. $\left.\mathcal{C}\left(r ; s_{1}, r-1\right)=\mathcal{C}\left(r ; s_{1}\right)\right)$. Therefore, when studying the set $\mathcal{C}\left(r ; s_{1}, \ldots, s_{l}\right)$, one may assume $l \geq 2$. Define

$$
G\left(r ; s_{1}, \ldots, s_{l}\right)=\max \left\{p_{a}(C): C \in \mathcal{C}\left(r ; s_{1}, \ldots, s_{l}\right)\right\},
$$

where $p_{a}(C)$ denotes the arithmetic genus of $C$. We refer to [CCD2] for a general discussion on the genus of curves verifying flag conditions and its relationship with Castelnuovo-Halphen theory. Improving Theorem 3.3 and Corollary 3.4 in [CCD2], in the present paper we prove the following:

Theorem. Assume that $s_{1} \gg \cdots \gg s_{l}$, and fix a curve $C \in \mathcal{C}\left(r ; s_{1}, \ldots, s_{l}\right)$ of maximal arithmetic genus $p_{a}(C)=G\left(r ; s_{1}, \ldots, s_{l}\right)$. Then one has:

(a) $C$ is arithmetically Cohen-Macaulay;

(b) there exists a unique flag

$$
C=V_{s_{1}}^{1} \subset V_{s_{2}}^{2} \subset \cdots \subset V_{s_{l}}^{l} \subset \mathbf{P}^{r}
$$

where $V_{s}^{j}$ denotes an integral projective subvariety of $\mathbf{P}^{r}$ of degree $s$ and dimension $j$;

(c) for any $i=1, \ldots, l$ one has

$$
V_{s_{i}}^{(1)} \in \mathcal{C}\left(r-i+1 ; s_{i}, \ldots, s_{l}\right) \quad \text { and } \quad p_{a}\left(V_{s_{i}}^{(1)}\right)=G\left(r-i+1 ; s_{i}, \ldots, s_{l}\right),
$$

where $V_{s_{i}}^{(1)}$ denotes the curve intersection of $V_{s_{i}}^{i}$ with a general linear subspace of $\mathbf{P}^{r}$ of dimension $r-i+1$;

(d) there exists a rational number $R=R\left(r ; s_{1}, \ldots, s_{l}\right)$ depending only on $r, s_{1}$, $\ldots, s_{l}$, such that

$$
G\left(r ; s_{1}, \ldots, s_{l}\right)=\frac{s_{1}^{2}}{2 s_{2}}+\frac{s_{1}}{2 s_{2}}\left[2 G\left(r-1 ; s_{2}, \ldots, s_{l}\right)-2-s_{2}\right]+R
$$

and $|R| \leq s_{2}^{3} /(r-2)$.

Properties (b) and (c) above show a sort of a hierarchical structure of the family of curves with maximal genus verifying flag conditions. Moreover, with the exception of the "constant term" $R$, property (d) gives a recurrence formula for $G\left(r ; s_{1}, \ldots, s_{l}\right)$. In Remark (iii) below, we make explicit what the condition $s_{1} \gg \cdots \gg s_{l}$ means.

We will prove the theorem using some of the results contained in [CCD2], and using induction on $l$, the case $l=2$ being contained in the main result of [CCD]. The induction argument relies on the following:

Lemma. Let $S \subset \mathbf{P}^{r}$ be an irreducible, reduced, nondegenerate projective surface, of degree $s \geq r-1 \geq 2$. Denote by $S^{(1)} \subset \mathbf{P}^{r-1}$ the general hyperplane section of $S$, by $\pi$ its arithmetic genus, by $\mathcal{I}_{S^{(1)}}$ its ideal sheaf in $\mathbf{P}^{r-1}$, by $S^{(0)}$ the general hyperplane section of $S^{(1)}$ and by $h_{S^{(0)}}$ its Hilbert function. For any integer $i$, denote by $\delta_{i}$ the dimension of the kernel of the natural map $H^{1}\left(\mathbf{P}^{r-1}, \mathcal{I}_{S^{(1)}}(i-1)\right) \rightarrow$ $H^{1}\left(\mathbf{P}^{r-1}, \mathcal{I}_{S^{(1)}}(i)\right)$.

Let $C \subset S$ be an irreducible, reduced, nondegenerate projective curve, of degree $d \geq s^{2}+s(r-4)^{2}$ for $3 \leq r \leq 4$, and of degree $d>s^{2}-s$ for $r \geq 5$. Denote by $p_{a}(C)$ the arithmetic genus of $C$, by $\Gamma$ the general hyperplane section of $C$, by $h_{\Gamma}$ its Hilbert function, and define $m$ and $\epsilon$ by dividing

$$
d-1=m s+\epsilon, \quad 0 \leq \epsilon \leq s-1 .
$$


Moreover define:

$R(C)=\frac{1+\epsilon}{2 s}(s+1-\epsilon-2 \pi)-\sum_{i=1}^{+\infty}(i-1)\left(s-h_{S^{(0)}}(i)\right)+\sum_{i=1}^{+\infty}(i-1) \delta_{i}+\sum_{i=m+1}^{+\infty}\left(d-h_{\Gamma}(i)\right)$.

Then one has:

$$
p_{a}(C) \leq \sum_{i=1}^{+\infty}\left(d-h_{\Gamma}(i)\right)=\frac{d^{2}}{2 s}+\frac{d}{2 s}(2 \pi-2-s)+R(C)
$$

and $|R(C)| \leq s^{3} /(r-2)$.

The proof of the lemma entirely relies on Castelnuovo theory. In particular we use the following general formula,

$$
\sum_{i=1}^{+\infty}\left(s-h_{S^{(0)}}(i)\right)=\pi+\sum_{i=1}^{+\infty} \delta_{i}
$$

(see [Ci], p. 31), which enables us to compute the coefficient of the linear term $d$ in $(2.1)$.

Notice that, when the surface $S \subset \mathbf{P}^{r}$ is smooth and subcanonical, using the Hodge Index Theorem and the adjunction formula, one has

$$
p_{a}(C) \leq \frac{d^{2}}{2 s}+\frac{d}{2 s}(2 \pi-2-s)+1,
$$

for any curve $C \subset S$. Therefore, one may interpret (2.1) as a "coarse numerical adjunction formula", which holds for integral projective curves on any integral projective surface $S \subset \mathbf{P}^{r}$.

On the other hand, by [CCD], the Main Theorem and Proposition 4.2, we know that on any such surface (when $d>s^{2}-s$ ) one has

$$
p_{a}(C) \leq \frac{d^{2}}{2 s}+\frac{d}{2 s}(2 G(r-1 ; s, r-2)-2-s)+R_{1},
$$

where $G(r-1 ; s, r-2)$ is the Castelnuovo bound for a nondegenerate curve of degree $s$ in $\mathbf{P}^{r-1}$, and $R_{1}$ is a rational number that depends only on $s, r$ and $\epsilon$ (for the exact definition of $R_{1}$ we refer to [CCD], pp. 230-231). On (certain) Castelnuovo surfaces (i.e. surfaces whose general hyperplane section is a Castelnuovo curve) the previous bound (0.1) is sharp (see [CCD], pp. 243-244). Now, in view of our lemma, we may refine the bound (0.1) proved in [CCD] in the following sense:

Corollary. Fix integers $r, s$ and $\pi$ such that $r \geq 3, s \geq r-1$ and $\pi \geq 0$. Let $C \subset \mathbf{P}^{r}$ be an integral, nondegenerate, projective curve of degree $d \gg s$. Assume that $C$ is not contained in any surface of degree $<s$, and not contained in any surface of degree $s$ with linear genus $>\pi$. Then one has:

$$
p_{a}(C) \leq \frac{d^{2}}{2 s}+\frac{d}{2 s}(2 \pi-2-s)+\frac{s^{3}}{r-2} .
$$

In Remark (iii) below, we make explicit the condition $d \gg s$.

Notice that the bound (3.1) given in the corollary is not sharp. However, dividing (3.1) by $d$, and assuming as before $d \gg s$, we get

$$
e(C) \leq \frac{d}{s}+\frac{2 \pi-2-s}{s},
$$


where $e(C)$ denotes the speciality index of $C$, i.e.

$$
e(C)=\max \left\{t \in \mathbf{Z}: H^{1}\left(C, \mathcal{O}_{C}(t)\right) \neq 0\right\}
$$

(recall that by $[\mathrm{GP}]$, p. 51 , Remarque 3.6, one has $e(C) d \leq 2 p_{a}(C)-2$ ). Now, at least in certain cases, the previous bound (0.2) is sharp (e.g. when $C$ is a complete intersection on a complete intersection surface of degree $s$ and linear genus $\pi$ ). The bound (0.2) should be compared with the "Théorème de spécialité" in [GP], p. 32. We have in mind to give more information on $(0.2)$ in a forthcoming paper.

Now we are going to prove the announced results. We work over the complex field and we use the standard notation of algebraic geometry. We begin by showing the lemma.

Proof of Lemma. In view of [GP] we may assume $r \geq 4$. For the proof of the inequality $p_{a}(C) \leq \sum_{i=1}^{+\infty}\left(d-h_{\Gamma}(i)\right)$ we refer to [EH], Corollary 3.2.

In order to compute the sum $\sum_{i=1}^{+\infty}\left(d-h_{\Gamma}(i)\right)$, first notice that by Bézout's theorem we have $h_{\Gamma}(i)=h_{S^{(1)}}(i)$ for any $i \leq m$, where $h_{S^{(1)}}$ denotes the Hilbert function of $S^{(1)}$. Hence we may write:

$$
\sum_{i=1}^{+\infty}\left(d-h_{\Gamma}(i)\right)=\sum_{i=1}^{m}\left(d-h_{S^{(1)}}(i)\right)+\sum_{i=m+1}^{+\infty}\left(d-h_{\Gamma}(i)\right) .
$$

From [Ci], p. 30, we know that

$$
\left(h_{S^{(1)}}(j)-h_{S^{(1)}}(j-1)\right)-h_{S^{(0)}}(j)=\delta_{j}
$$

for any integer $j$. Therefore we have:

$$
\begin{gathered}
\sum_{i=1}^{m}\left(d-h_{S^{(1)}}(i)\right)=\sum_{i=1}^{m}\left[d-\sum_{j=0}^{i}\left(h_{S^{(1)}}(j)-h_{S^{(1)}}(j-1)\right)\right]= \\
\sum_{i=1}^{m}\left[d-\sum_{j=0}^{i}\left(h_{S^{(0)}}(j)+\delta_{j}\right)\right]=m d-\left[m\left(1+\delta_{0}\right)+\sum_{i=1}^{m}(m-i+1)\left(h_{S^{(0)}}(i)+\delta_{i}\right)\right]= \\
m(d-1)-\sum_{i=1}^{m}(m-i+1)\left(s-s+h_{S^{(0)}}(i)+\delta_{i}\right)= \\
\frac{m s}{2}(m-1)+m \epsilon+m \sum_{i=1}^{m}\left(s-h_{S^{(0)}}(i)-\delta_{i}\right)-\sum_{i=1}^{m}(i-1)\left(s-h_{S^{(0)}}(i)-\delta_{i}\right) .
\end{gathered}
$$

Now define $w$ and $v$ by dividing

$$
s-1=w(r-2)+v, \quad 0 \leq v \leq r-3 .
$$

By [EH, Theorem 3.7] and [GLP], we know that

$$
h_{S^{(0)}}(i)=s \quad \text { for any } i \geq w+1
$$

and

$$
H^{1}\left(\mathbf{P}^{r-1}, \mathcal{I}_{S^{(1)}}(i)\right)=0 \quad \text { for any } i \geq s-r+2 .
$$


Since $d \gg s$, then $m \geq w+1$ and $m \geq s-r+2$, and so, taking into account [Ci], p. 31, we get

$$
\sum_{i=1}^{m}\left(s-h_{S^{(0)}}(i)-\delta_{i}\right)=\sum_{i=1}^{+\infty}\left(s-h_{S^{(0)}}(i)-\delta_{i}\right)=\pi
$$

and

$$
\sum_{i=1}^{m}(i-1)\left(s-h_{S^{(0)}}(i)-\delta_{i}\right)=\sum_{i=1}^{+\infty}(i-1)\left(s-h_{S^{(0)}}(i)-\delta_{i}\right) .
$$

Continuing the previous computation, we have

$$
\sum_{i=1}^{m}\left(d-h_{S^{(1)}}(i)\right)=\frac{m s}{2}(m-1)+m \epsilon+m \pi-\sum_{i=1}^{+\infty}(i-1)\left(s-h_{S^{(0)}}(i)-\delta_{i}\right) .
$$

Replacing $m$ with $(d-1-\epsilon) / s$, and taking into account (2.2), we get (2.1).

To conclude the proof of the lemma we have to estimate $R(C)$. We will analyze each of the four terms appearing in the definition of $R(C)$.

First notice that

$$
-\pi \leq \frac{1+\epsilon}{2 s}(s+1-\epsilon-2 \pi) \leq \frac{s+1}{2} .
$$

We may estimate the arithmetic genus $\pi$ of $S^{(1)}$ using the Castelnuovo bound for curves of degree $s$ in $\mathbf{P}^{r-1}$ (compare with (2.3)):

$$
\pi \leq\left(\begin{array}{c}
w \\
2
\end{array}\right)(r-2)+w v \leq \frac{s^{2}}{2(r-2)},
$$

from which we obtain

$$
-\frac{s^{2}}{2(r-2)} \leq \frac{1+\epsilon}{2 s}(s+1-\epsilon-2 \pi) \leq \frac{s+1}{2} .
$$

Now we turn to the next term. From $[\mathrm{EH}]$ we know that

$$
h_{S^{(0)}}(i) \geq \min \{s, i(r-2)+1\}
$$

for any $i \geq 1$. Since

$$
\sum_{i=1}^{+\infty}(i-1)(s-\min \{s, i(r-2)+1\})=\left(\begin{array}{c}
w \\
3
\end{array}\right)(r-2)+\left(\begin{array}{c}
w \\
2
\end{array}\right) v \leq \frac{s^{3}}{3(r-2)^{2}},
$$

it follows that

$$
0 \leq \sum_{i=1}^{+\infty}(i-1)\left(s-h_{S^{(0)}}(i)\right) \leq \frac{s^{3}}{3(r-2)^{2}}
$$

In order to estimate the third term, first notice that from (2.4) we have

$$
\sum_{i=1}^{+\infty}(i-1) \delta_{i}=\sum_{i=1}^{s}(i-1) \delta_{i} \leq(s-1) \sum_{i=1}^{+\infty} \delta_{i} .
$$


On the other hand, from [Ci, p. 31] and (2.6), we have

$$
\begin{aligned}
\sum_{i=1}^{+\infty} \delta_{i} & \leq \sum_{i=1}^{+\infty}\left(s-h_{S^{(0)}}(i)\right) \\
& \leq \sum_{i=1}^{+\infty}(s-\min \{s, i(r-2)+1\})=\left(\begin{array}{c}
w \\
2
\end{array}\right)(r-2)+w v \leq \frac{s^{2}}{2(r-2)} .
\end{aligned}
$$

Putting all together we get

$$
0 \leq \sum_{i=1}^{+\infty}(i-1) \delta_{i} \leq \frac{s^{2}(s-1)}{2(r-2)} .
$$

Finally we are going to analyze the last term. From [CCD, Propositions 4.1 and $4.2]$, we know that

$$
\sum_{i=m+1}^{+\infty}\left(d-h_{\Gamma}(i)\right)=\sum_{i=m+1}^{m+w}\left(d-h_{\Gamma}(i)\right) .
$$

Since $h_{\Gamma}(i) \geq h_{\Gamma}(m)$ for $i \geq m$, we deduce that

$$
\sum_{i=m+1}^{+\infty}\left(d-h_{\Gamma}(i)\right) \leq w\left(d-h_{\Gamma}(m)\right) .
$$

Since $d \gg s$, by Bézout's theorem and (2.4) we have

$$
h_{\Gamma}(m)=h_{S^{(1)}}(m)=h^{0}\left(S^{(1)}, \mathcal{O}_{S^{(1)}}(m)\right) .
$$

On the other hand, from (2.6) we deduce

$$
h^{1}\left(\mathbf{P}^{r-2}, \mathcal{I}_{S^{(0)}}(i)\right)=0
$$

for any $i \geq w+1\left(\mathcal{I}_{S^{(0)}}=\right.$ ideal sheaf of $S^{(0)}$ in $\left.\mathbf{P}^{r-2}\right)$. This implies that

$$
h^{1}\left(S^{(1)}, \mathcal{O}_{S^{(1)}}(m)\right)=0 .
$$

From (2.10) it follows that

$$
h_{\Gamma}(m)=m s+1-\pi .
$$

From Castelnuovo's bound on $\pi$ we deduce

$$
w\left(d-h_{\Gamma}(m)\right)=w(\epsilon+\pi) \leq \frac{s^{3}}{2(r-2)^{2}},
$$

and so, from (2.9), we get

$$
0 \leq \sum_{i=m+1}^{+\infty}\left(d-h_{\Gamma}(i)\right) \leq \frac{s^{3}}{2(r-2)^{2}} .
$$

Using (2.5), (2.7), (2.8) and (2.11), we obtain the estimate for $|R(C)|$. This concludes the proof of the lemma.

Next we give the proof of the theorem. 
Proof of Theorem. The case $l=2$ is contained in the main result of [CCD]. Therefore we may argue by induction on $l$, and assume $l \geq 3$.

For the existence of the flag (1.1) we refer to [CCD2, Corollary 2.8]. The uniqueness follows by Bézout's theorem and the assumption $s_{1} \gg \cdots \gg s_{l}$. This proves property (b).

By the lemma we know that

$$
p_{a}(C) \leq \frac{s_{1}^{2}}{2 s_{2}}+\frac{s_{1}}{2 s_{2}}\left(2 \pi-2-s_{2}\right)+s_{2}^{3} /(r-2),
$$

where $\pi$ denotes the linear arithmetic genus of $V_{s_{2}}^{2}$. Since $s_{2} \gg \cdots \gg s_{l}$, then by Bézout's theorem we have $V_{s_{2}}^{(1)} \in \mathcal{C}\left(r-1 ; s_{2}, \ldots, s_{l}\right)$, and so

$$
\pi \leq G\left(r-1 ; s_{2}, \ldots, s_{l}\right) .
$$

Now fix a curve $D \in \mathcal{C}\left(r-1 ; s_{2}, \ldots, s_{l}\right)$ of maximal arithmetic genus. By induction, this curve is arithmetically Cohen-Macaulay and determines a flag

$$
D=W_{s_{2}}^{1} \subset W_{s_{3}}^{2} \subset \cdots \subset W_{s_{l}}^{l-1} \subset \mathbf{P}^{r-1} .
$$

Since $s_{1} \gg s_{2}$, by [CCD2, Lemma 2.6], we may construct on the cone $C(D)$ over $D$ in $\mathbf{P}^{r}$, an integral, nondegenerate, projective and arithmetically Cohen-Macaulay curve $E \subset C(D)$ of degree $s_{1}$. $E$ lies on the cone of the flag (1.4); therefore $E \in \mathcal{C}\left(r ; s_{1}, s_{2}, \ldots, s_{l}\right)$. Moreover, since the general hyperplane section of $C(D)$ has arithmetic genus $G\left(r-1 ; s_{2}, \ldots, s_{l}\right)$ and $E$ is arithmetically Cohen-Macaulay, then by [EH, Remark 3.1.1] and our lemma, we have

$$
p_{a}(E)=\sum_{i=1}^{+\infty}\left(s_{1}-h_{E^{\prime}}(i)\right)=\frac{s_{1}^{2}}{2 s_{2}}+\frac{s_{1}}{2 s_{2}}\left[2 G\left(r-1 ; s_{2}, \ldots, s_{l}\right)-2-s_{2}\right]+R_{2}
$$

with $\left|R_{2}\right| \leq s_{2}^{3} /(r-2)$ ( $h_{E^{\prime}}=$ the Hilbert function of the general hyperplane section $E^{\prime}$ of $\left.E\right)$. Since $p_{a}(E) \leq G\left(r ; s_{1}, \ldots, s_{l}\right)=p_{a}(C)$ and $\left|R_{2}\right| \leq s_{2}^{3} /(r-2)$, and since $s_{1} \gg s_{2}$, then from (1.2), (1.3) and (1.5) we get

$$
\pi=G\left(r-1 ; s_{2}, \ldots, s_{l}\right) .
$$

This means that $V_{s_{2}}^{(1)}$ is a curve of maximal genus verifying the flag condition $\left(r-1 ; s_{2}, \ldots, s_{l}\right)$. This proves, by induction, property (c).

In particular, $V_{s_{2}}^{(1)}$ is arithmetically Cohen-Macaulay. Hence, using again [CCD2, Lemma 2.6], we may construct an arithmetically Cohen-Macaulay curve $F$ belonging to $\mathcal{C}\left(r ; s_{1}, \ldots, s_{l}\right)$, whose general hyperplane section $F^{\prime}$ has the same Hilbert function as the general hyperplane section $C^{\prime}$ of $C$. It follows that

$$
\begin{aligned}
G\left(r ; s_{1}, \ldots, s_{l}\right)=p_{a}(C) \leq \sum_{i=1}^{+\infty}\left(s_{1}-h_{C^{\prime}}(i)\right) \\
=\sum_{i=1}^{+\infty}\left(s_{1}-h_{F^{\prime}}(i)\right)=p_{a}\left(F^{\prime}\right) \leq G\left(r ; s_{1}, \ldots, s_{l}\right),
\end{aligned}
$$

and so

$$
p_{a}(C)=\sum_{i=1}^{+\infty}\left(s_{1}-h_{C^{\prime}}(i)\right)
$$

i.e., $C$ is arithmetically Cohen-Macaulay. This proves property (a). 
At this point, taking into account that $C \subset V_{s_{2}}^{2}$, property (d) follows from (1.7) and the previous lemma. This concludes the proof of the theorem.

Finally we turn to the proof of the corollary.

Proof of Corollary. If $C$ is not contained in any surface of degree $<s+1$, then, from the main result of $[\mathrm{CCD}]$ and our Lemma (compare with (0.1)), we deduce that

$$
p_{a}(C) \leq \frac{d^{2}}{2(s+1)}+\frac{d}{2(s+1)}(2 G(r-1 ; s+1, r-2)-2-(s+1))+\frac{(s+1)^{3}}{r-2},
$$

where $G(r-1 ; s+1, r-2)$ is the Castelnuovo bound for a nondegenerate curve of degree $s+1$ in $\mathbf{P}^{r-1}$. Since $d \gg s$, the previous bound (3.2) is strictly less than the bound appearing in (3.1). Therefore we may assume that $C$ is contained on some surface of degree $s$, with linear genus $\leq \pi$. In this case the corollary follows from the lemma. This concludes the proof of the corollary.

Remark. i) With the same notation as in the lemma, we notice that when the surface $S$ is arithmetically Cohen-Macaulay, then all $\delta_{i}$ vanish and $\sum_{i=1}^{+\infty}(i-1)\left(s-h_{S^{(0)}}(i)\right)$ is equal to the arithmetic genus $p_{a}(S)$ of $S$ (see [D], Remark 2.3). Therefore, in this case, we have

$$
R(C)=\frac{1+\epsilon}{2 s}(s+1-\epsilon-2 \pi)-p_{a}(S)+\sum_{i=m+1}^{+\infty}\left(d-h_{\Gamma}(i)\right) .
$$

In particular, in the theorem, since we know that the surface $V_{s_{2}}^{2}$ is arithmetically Cohen-Macaulay, we have

$$
R=\frac{1+\epsilon}{2 s}\left(s+1-\epsilon-2 G\left(r-1 ; s_{2}, \ldots, s_{l}\right)\right)-p_{a}\left(V_{s_{2}}^{2}\right)+\sum_{i=m+1}^{+\infty}\left(d-h_{\Gamma}(i)\right),
$$

where $h_{\Gamma}$ is the Hilbert function of the general hyperplane section of any maximal curve $C \in \mathcal{C}\left(r ; s_{1}, \ldots, s_{l}\right)$.

(ii) Again in the lemma, we notice that when $S$ is a Castelnuovo surface, using the main result of $[\mathrm{CCD}]$, one may prove that

$$
R(C)=O\left(s^{2}\right) .
$$

(iii) In proving the theorem, we need the numerical assumption $s_{1} \gg \cdots \gg s_{l}$ only to use Corollary 2.8 in [CCD2], Bézout's theorem, and to prove (1.6). To this purpose, it suffices to assume, for any $i=1, \ldots, l-1$,

$$
\begin{gathered}
s_{i} \geq 8(l-1)\left[(l-i+1)^{2}+2(l-i+1)+9\right] \frac{\left(s_{i+1}+1\right)^{3}}{r-i-1}, \\
s_{i}>\frac{\left(s_{i+1}+1\right)^{2}}{r-i-1}+(2 r-2)\left(s_{i+1}+1\right), \\
s_{i}>2 \frac{\left(s_{i+1}+1\right)}{r-i-1} \prod_{j=1}^{r-1-i}\left[(r-i) !\left(s_{i+1}+1\right)\right]^{\frac{1}{r-i-j}}, \quad \text { and } \\
s_{i}>2 \frac{s_{i+1}^{4}}{r-i-1} .
\end{gathered}
$$


We also make explicit the numerical assumption $d \gg s$ made in the corollary. In fact, we only need it for using [CCD], and to compare (3.2) with (3.1). To this aim, it suffices to assume

$$
d>\frac{2(s+1)}{r-2} \prod_{i=1}^{r-2}[(r-1) !(s+1)]^{\frac{1}{r-1-i}} \quad \text { and } \quad d>\frac{6(s+1)^{3}}{r-2} .
$$

\section{REFERENCES}

[C] G. Castelnuovo, Ricerche di geometria sulle curve algebriche, Zanichelli, Bologna (1937).

[CCD] L. Chiantini, C. Ciliberto and V. Di Gennaro, The genus of projective curves, Duke Math. J. 70/2 (1993), 229-245. MR1219813 (94b:14027)

[CCD2] L. Chiantini, C. Ciliberto and V. Di Gennaro, On the genus of projective curves verifying certain flag conditions, Boll. U.M.I. (7) 10-B (1996), 701-732. MR1411524 (98g:14028)

[Ci] C. Ciliberto, Hilbert functions on finite sets of points and the genus of a curve in a projective space, in Space Curves: Proceedings, Rocca di Papa, 1985, Lecture Notes in Math., Springer-Verlag, Berlin 1266, 24-73. MR908707 (89c:14039)

[D] V. Di Gennaro, A bound on the geometric genus of projective varieties verifying certain flag conditions, Trans. Amer. Math. Soc. 349 (3) (1997), 1121-1151. MR1390976 (97f:14043)

[EH] D. Eisenbud and J. Harris, Curves in Projective Space, Sém. Math. Sup. 85, Les Presses du l'Université de Montréal, Montréal (1982). MR685427 (84g:14024)

[GLP] L. Gruson, R. Lazarsfeld and C. Peskine, On a theorem of Castelnuovo and the equations defining space curves, Invent. Math. 72 (1983), 491-506. MR704401 (85g:14033)

[GP] L. Gruson and C. Peskine, Genre des courbes dans l'espace projectif, Algebraic Geometry: Proceedings, Norway, 1977, Lecture Notes in Math., Springer-Verlag, New York 687 (1978), 31-59. MR527229 (81e:14019)

[H] G. Halphen, Mémoire sur la classification des courbes gauches algébriques, Oeuvres Complètes, vol. III; also J. École Polytechnique 52 (1882), 1-200.

[N] M. Noether, Zur Grundlegung der Theorie der algebraischen Raumcurven, Königlichen Akad. der Wissenschaften (1883).

Dipartimento di Matematica, Università di Roma Tor Vergata, Via della Ricerca SCIENTIFICA, 00133 Roma, ItAlia

E-mail address: digennar@axp.mat.uniroma2.it 